\title{
Phosphoribosyl Pyrophosphate Amido Transferase: A New Prognostic Biomarker for Hepatocellular Carcinoma
}

\section{Qingfei Chu* \\ Xinyu Gu* \\ Qiuxian Zheng \\ Jing Wang \\ Haihong Zhu (DD}

State Key Laboratory for Diagnosis and Treatment of Infectious Diseases, National Clinical Research Center for Infectious Diseases, Collaborative Innovation Center for Diagnosis and Treatment of Infectious Diseases, The First Affiliated Hospital, College of Medicine, Zhejiang University, Hangzhou, Zhejiang, 310003, People's Republic of China

*These authors contributed equally to this work
Correspondence: Haihong Zhu

Tel +86-13656632056

Email zhuhh72@zju.edu.cn
Background: PPAT (phosphoribosyl pyrophosphate amido transferase) catalyzes the first committed step of de novo purine biosynthesis and is a key regulatory point in the biosynthesis of nascent purine nucleotides. However, the clinical significance and biologic role of PPAT in hepatocellular carcinoma (HCC) remain unknown.

Methods: We compared the expression of PPAT in carcinomatous and precancerous hepatocellular carcinoma tissues by immunohistochemistry in 90 cases of HCC. Correlation analysis was also made on clinical data, survival, classification, and staging.

Results: The expression of PPAT in HCC tumor tissues is significantly higher than that in adjacent normal tissues. The results of the Kaplan-Meier analysis showed that HCC patients with high PPAT expression survived shorter than those with low PPAT expression. Moreover, the expression of PPAT was significantly associated with the tumor grade $(\mathrm{P}=0.014)$, PD-L1 $(\mathrm{P}<0.001)$, and CTLA4 $(\mathrm{P}=0.003)$. The later grade of the tumor, the higher the expression of PPAT. In the PD-L1 high expression group, PPAT is also highly expressed.

Conclusion: Our study demonstrated that PPAT expression might be included in the process of carcinogenesis and prognosis. Hence, PPAT could be served as a new prognostic biomarker for patients of HCC.

Keywords: PPAT, HCC, prognosis, biomarker, metabolism

\section{Introduction}

Liver cancer remains a global health challenge, with an estimated incidence of 1 million cases by $2025 .{ }^{1}$ Hepatocellular carcinoma (HCC) is the most common form of liver cancer, accounting for approximately $90 \%$ of cases. ${ }^{1}$ According to global cancer statistics, HCC is the third leading cause of cancer death. ${ }^{2}$ Standard treatment for HCC includes surgical resection, transarterial embolization, radiation therapy, and chemotherapy. However, these treatments are often inadequate and eventually lead to the development of advanced HCC. Therefore, studying new treatments of HCC is necessary for understanding HCC mechanisms and improving the prognosis of HCC.

Phosphoribosyl pyrophosphate amido transferase (PPAT) is a key rate-limiting reaction in purine biosynthesis, transferring gamma-nitrogen from glutamine to 5 phosphoribosyl pyrophosphate (PRPP). ${ }^{3}$ In the malignant development of cancer, carbon and nitrogen metabolism has changed. The fate of glutamine nitrogen is controlled by glutaminase (GLS1) and PPAT, shifting from the senescence pathway to the TCA cycle to nucleotide biosynthesis. ${ }^{4}$ Several studies have identified PPAT is related to breast cancer, ${ }^{5}$ lung cancer, ${ }^{6}$ prostate cancer, ${ }^{7}$ colorectal carcinoma, ${ }^{8}$ 
and hepatocellular carcinoma. ${ }^{9}$ These results show PPAT may be a significant target of therapy.

In this study, we compared the expression of PPAT in liver cancer tissues and control tissues and explored the relationship between its expression and clinical parameters, as well as its possible clinical value.

\section{Materials and Methods \\ Clinical Tissues Specimens}

We constructed a tissue microarray (TMA) comprised of 90 HCC tissues and 90 normal liver tissues, all of them were purchased from Outdo Biotechnology Company (Shanghai, China). All patient data that were used in the present study had complete clinical information, including age, sex, grade, TNM stage, and survival time.

\section{Immunohistochemistry (IHC) Analysis}

The tissue chips were placed in an oven at $63^{\circ} \mathrm{C}$ and waxed for one hour. And then dewax is completed in a fully automatic dye machine. Dewaxed in xylene, rehydrated through graded alcohol. TMA slides were incubated with PPAT primary antibody (dilution 1:25, HPA036091, Atlas Antibodies $\mathrm{AB}$ ) at $4^{\circ} \mathrm{C}$ overnight. The slides were removed from the refrigerator, rewarmed for 45 minutes at room temperature, and washed with PBST buffer. The slides were put into the DAKO all-active immunity histochemical staining system, and the corresponding programs were selected to run blocking, secondary antibody binding, and $\mathrm{DAB}$ coloration. Hematoxylin is then stained for one minute, immersed in $0.25 \%$ hydrochloric acid alcohol for not less than two seconds, and rinsed with tap water for more than two minutes. The slides were dried at room temperature and sealed with neutral resin.

\section{Evaluation of Immunostaining Intensity}

Based on the intensity of PPAT staining and the degree of positive cells, the scores of immunohistochemical tissues were independently evaluated by three experienced senior pathologists who did not know the characteristics of the patient in advance. The staining intensity: scored 0 (negative), scored $1(+)$, scored $2(++)$, and scored $3(+++)$. Then the rats were grouped according to the total score which was the product of "staining intensity score" and "staining positive rate". We defined the score $<2$ as the low expression of PPAT and the score $\geq 2$ as the high expression of PPAT.

\section{Statistical Analysis}

We performed a one-way analysis of variance and unpaired two-tailed $t$-test for comparisons. ROC curve analysis was used to determine the critical value of PPAT high expression. Kaplan-Meier method was used for the survival curve. P-values less than 0.05 were considered statistically significant.

\section{Results}

\section{PPAT Expression Was Elevated in HCC}

\section{Tumor Tissue}

The expression level of PPAT in 90 cases of HCC and 90 cases of normal liver tissues adjacent to cancer was evaluated by tissue microarray. Immunohistochemical staining showed that the immune response of PPAT mainly appeared in the cytoplasm of tumor cells. The PPAT expression in HCC tumor tissues was higher than that in adjacent normal tissues $(\mathrm{P}<0.001)$ (Figure 1).

\section{The Relationship Between the PPAT Level and Clinicopathologic Characteristics of $\mathrm{HCC}$}

In order to further understand the relationship between PPAT and HCC, we conducted further research. Then we displayed the clinicopathological characteristics in Table 1. The expression of PPAT was significantly correlated with three parameters, including tumor grade $(\mathrm{P}=0.014)$, PD-L1 $(\mathrm{P}<0.001)$, and CTLA4 $(\mathrm{P}=0.003)$. This is consistent with the previous study of our group, ${ }^{9}$ PPAT is related to immunosuppression, suggesting that PPAT can be used as a predictive target in immunotherapy. We also found that there was no significant association among PPAT expression and age, sex, TNM stage, liver cirrhosis, alpha-fetoprotein (AFP) levels, or alanine transaminase (ALT).

\section{The Correlation Between High Expression of PPAT and Low Survival Rate in $\mathrm{HCC}$}

In addition, we evaluated the association of PPAT expression with survival in patients with HCC. Kaplan-Meier 

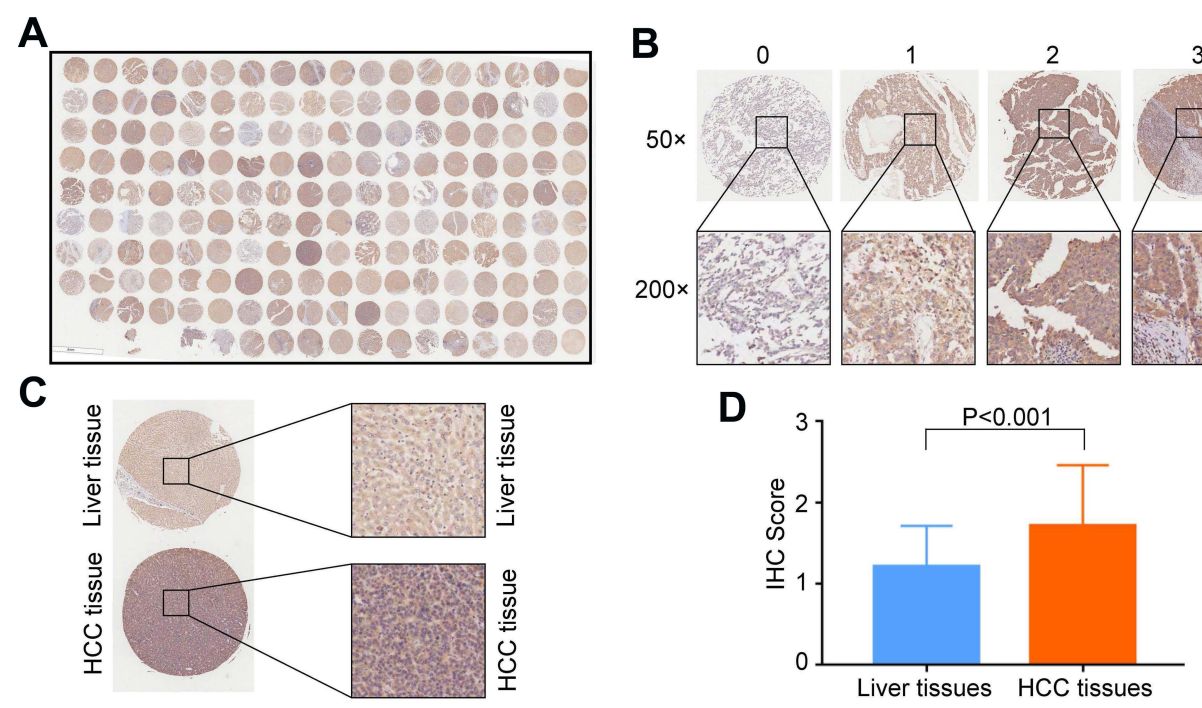

Figure I PPAT is overexpressed in HCC tissue. (A) Panoramic scanning for HCC TMA. (B) Representative PPAT and immunohistochemical staining patterns with different staining scores in HCC tissues. (C) Representative PPAT staining in HCC tissues and normal samples. (D) Histological scoring of PPAT in HCC tissues and normal samples.

survival analysis curve and log-rank statistical test show that patients with high PPAT expression may have significantly shorter overall survival $(\mathrm{P}=0.023)$ (Figure 2A), disease-free survival $(\mathrm{P}=0.007)$ (Figure $2 \mathrm{~B})$.

\section{Discussion}

The incidence of HCC is increasing worldwide and the mortality rate of advanced $\mathrm{HCC}$ is high. ${ }^{10}$ Therapeutic approaches such as local excision, chemoradiotherapy, and immunosuppressive therapy have been improved, but recurrence of therapeutic resistance leads to higher mortality. The recurrence rate after 5 years is as high as $60 \%-70 \%{ }^{11}$ Therefore, new biomarkers and effective therapeutic targets must be found as soon as possible. There have been a variety of studies in recent years. For example, $\mathrm{Lu}$ et al showed that lnc-TSPAN12 may contribute to the progression of $\mathrm{HCC}$ and thus provide a new diagnostic/prognostic biomarker. ${ }^{12}$ Studies have also found that in hepatocellular carcinoma tissues, the expression of HAND2-AS1 is regulated, and it promotes the development of tumors. The abnormal expression of HAND2-AS1 is closely related to tumor progression and prognosis. ${ }^{13}$ In addition, a large number of studies have provided new insights for improving therapeutic strategies for HCC by establishing multi-gene prognostic models for predicting the prognosis of patients with HCC. ${ }^{14,15}$
PPAT is the key step in the catalytic synthesis of purines. ${ }^{16}$ Robust metabolism is a hallmark of cancer, so PPAT could be an attractive and credible drug target for cancer treatment. ${ }^{17}$ Studies have shown that PPAT is involved in the occurrence and development of some tumors and can be used as a biomarker for the treatment and prognosis of these cancers. For example, PPAT regulates the proliferation, migration, and invasion of thyroid cancer cells by activating PKM2, ERK, and STAT3. ${ }^{7}$ Glutamine-mediated induction of PPAT and PAICS is an important metabolic event for the increased expression of these genes in lung tumorigenesis, thereby enhancing tumor-specific pyruvate kinase activity. ${ }^{6}$ In addition, PPAT has a strong correlation with small cell lung cancer (SCLC), and the consumption of PPAT inhibits the growth of SCLC. ${ }^{4} \mathrm{Zhu}$ et al found that PPAT is a direct estrogen receptor $\alpha(E R \alpha)$ target that is crucial for the survival and growth of breast cancer cells. ${ }^{5}$ However, there is no study on the correlation between liver cancer and PPAT.

Our study found that PPAT can be used as a prognostic biomarker for HCC. His high expression predicts a poor prognosis for patients with HCC. In addition, we also found that the expressions of PD-L1 (programmed death-ligand protein-1) and CTLA4 (cytotoxic T lymphocyte antigen-4) were up-regulated in HCC tumor tissues. Both are immunotherapeutic-related 
Table I The Relationship Between PPAT Expression and Clinicopathologic Parameters of Patients with HCC

\begin{tabular}{|c|c|c|c|c|c|c|}
\hline & \multirow[t]{2}{*}{ Variables } & \multicolumn{2}{|c|}{ PPAT Expression } & \multirow[t]{2}{*}{ Total } & \multirow[t]{2}{*}{$\chi^{2}$} & \multirow[t]{2}{*}{$P$ value } \\
\hline & & Low & High & & & \\
\hline \multirow[t]{3}{*}{ Age (years) } & & & & & 0.953 & 0.329 \\
\hline & $\leq 50$ & 16 & 22 & 38 & & \\
\hline & $>50$ & 16 & 34 & 50 & & \\
\hline \multirow[t]{3}{*}{ Sex } & & & & & 0.009 & 0.924 \\
\hline & Female & 3 & 7 & 10 & & \\
\hline & Male & 29 & 49 & 78 & & \\
\hline \multirow[t]{3}{*}{ Grade } & & & & & 6.091 & 0.014 \\
\hline & $1 / 2$ & 23 & 25 & 48 & & \\
\hline & 3 & 9 & 31 & 40 & & \\
\hline \multirow[t]{3}{*}{ T stage } & & & & & 0.763 & 0.382 \\
\hline & TI & 24 & 37 & 61 & & \\
\hline & $\mathrm{T} 2 / \mathrm{T} 3$ & 8 & 19 & 27 & & \\
\hline \multirow[t]{3}{*}{ TNM stage } & & & & & 0.763 & 0.382 \\
\hline & I & 24 & 37 & 61 & & \\
\hline & $\| / / I I$ & 8 & 19 & 27 & & \\
\hline \multirow[t]{3}{*}{$\mathrm{HBsAg}$} & & & & & 2.626 & 0.105 \\
\hline & Negative & 10 & 9 & 19 & & \\
\hline & Positive & 22 & 46 & 68 & & \\
\hline \multirow[t]{3}{*}{$\mathrm{HBcAb}$} & & & & & 0.012 & 0.912 \\
\hline & Negative & 2 & 5 & 7 & & \\
\hline & Positive & 30 & 48 & 78 & & \\
\hline \multirow[t]{3}{*}{ AFP } & & & & & 0.031 & 0.86 \\
\hline & Negative & 14 & 23 & 37 & & \\
\hline & Positive & 18 & 32 & 50 & & \\
\hline \multirow[t]{3}{*}{ ALT } & & & & & 0.63 & 0.427 \\
\hline & Negative & 17 & 34 & 51 & & \\
\hline & Positive & 15 & 21 & 36 & & \\
\hline \multirow[t]{3}{*}{ PD-LI } & & & & & 19.198 & 0.001 \\
\hline & Negative & 17 & 6 & 23 & & \\
\hline & Positive & 13 & 46 & 59 & & \\
\hline \multirow[t]{3}{*}{ CTLA4 } & & & & & 8.64 & 0.003 \\
\hline & Negative & 11 & 6 & 17 & & \\
\hline & Positive & 17 & 47 & 64 & & \\
\hline
\end{tabular}

Abbreviations: HCC, hepatocellular carcinoma; TNM, tumor node metastasis; AFP, alpha-fetoprotein; ALT, alanine transaminase; PD-LI, programmed death-ligand I; CTLA4, cytotoxic T-lymphocyte-associated protein 4.

factors that show potent immunomodulatory effects. ${ }^{18,19}$ Our study suggested that PPAT might be involved in immunosuppression, and it could be used to predict the effect of immunotherapy, which deserves further investigation. We believe that the use of PPAT to predict the prognosis of HCC has great benefits but also has some disadvantages. The advantage is that as long as one index is detected, the prognosis of liver cancer can be predicted, the method is simple and convenient, and the clinical promotion is facilitated. However, the disadvantages lie in the fact that it is difficult to obtain materials, tissue is needed, and only postoperative patients can be predicted. Therefore, further research is needed to realize the multifunctional and more convenient detection of the level of PPAT so as to judge the prognosis of liver cancer. For example, we can assess the overall expression of PPAT in blood by measuring its expression level.

\section{Conclusion}

In conclusion, our study demonstrates that a high level of PPAT is related to HCC prognosis, and the progression of HCC can be controlled by decreasing PPAT expression. However, we need more fundamental experiments to identify the molecular mechanism of PPAT in HCC progression. 

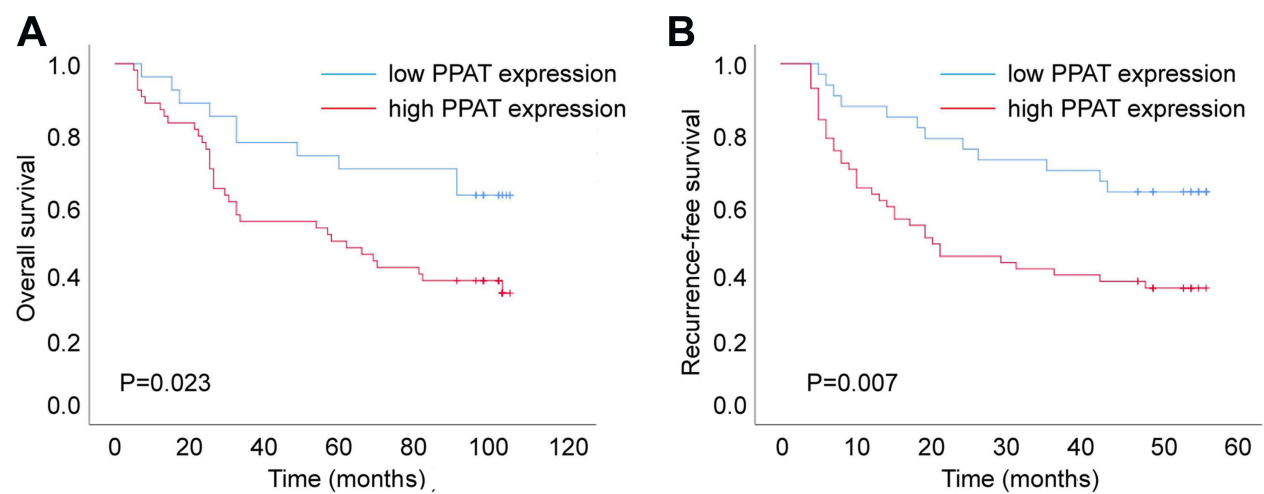

Figure 2 Kaplan-Meier survival analysis of PPAT expression in patients with HCC (Log Rank Test). (A) Overall survival and (B) recurrence-free survival analysis of the expression of PPAT in patients with HCC.

\section{Abbreviations}

PPAT, phosphoribosyl pyrophosphate amido transferase; HCC, hepatocellular carcinoma; PRPP, 5- phosphoribosyl pyrophosphate; GLS1, glutaminase; TMA, tissue microarray; IHC, immunohistochemistry; AFP, alpha-fetoprotein; ALT, alanine transaminase; SCLC, small cell lung cancer; ER $\alpha$, estrogen receptor $\alpha$; PD-L1, programmed deathligand protein-1; CTLA4, cytotoxic $\mathrm{T}$ lymphocyte antigen-4.

\section{Data Sharing Statement}

All data generated or analyzed during this study are included in this published article.

\section{Ethics Approval and Consent to Participate}

The study has been approved by the Ethics Committee of the First Affiliated Hospital of Zhejiang University.

\section{Funding}

This study was supported by grants awarded by the National Science and Technology Major Project of China (NO 2018ZX10302206), Science and Technology Major Projects of Zhejiang Province (NO 2018C04016), and the Science and Technology Major Projects of Ningbo (NO 2016C51008).

\section{Disclosure}

The authors declare that they have no competing interests.

\section{References}

1. Llovet JM, Kelley RK, Villanueva A, et al. Hepatocellular carcinoma. Nat Rev Dis Primers. 2021;7(1):6. doi:10.1038/s41572-020-00240-3
2. Jiang Y, Sun A, Zhao Y, et al. Proteomics identifies new therapeutic targets of early-stage hepatocellular carcinoma. Nature. 2019;567 (7747):257-261. doi:10.1038/s41586-019-0987-8

3. Cunningham JT, Moreno MV, Lodi A, et al. Protein and nucleotide biosynthesis are coupled by a single rate-limiting enzyme, PRPS2, to drive cancer. Cell. 2014;157(5):1088-1103. doi:10.1016/j.cell.2 014.03.052

4. Kodama M, Oshikawa K, Shimizu H, et al. A shift in glutamine nitrogen metabolism contributes to the malignant progression of cancer. Nat Commun. 2020;11(1):1320. doi:10.1038/s41467-02015136-9

5. Zhu D, Zhao Z, Cui G, et al. Single-cell transcriptome analysis reveals estrogen signaling coordinately augments one-carbon, polyamine, and purine synthesis in breast cancer. Cell Rep. 2018;25 (8):2285-2298.e2284. doi:10.1016/j.celrep.2018.10.093

6. Goswami MT, Chen G, Chakravarthi BV, et al. Role and regulation of coordinately expressed de novo purine biosynthetic enzymes PPAT and PAICS in lung cancer. Oncotarget. 2015;6(27):23445-23461. doi:10.18632/oncotarget.4352

7. Liu B, Song M, Qin H, et al. Phosphoribosyl pyrophosphate amidotransferase promotes the progression of thyroid cancer via regulating pyruvate kinase M2. Onco Targets Ther. 2020;13:7629-7639. doi:10.2147/ott.S253137

8. Kunicka T, Prochazka P, Krus I, et al. Molecular profile of 5-fluorouracil pathway genes in colorectal carcinoma. BMC Cancer. 2016;16(1):795. doi:10.1186/s12885-016-2826-8

9. Gu X, Guan J, Xu J, et al. Model based on five tumour immune microenvironment-related genes for predicting hepatocellular carcinoma immunotherapy outcomes. J Transl Med. 2021;19(1):26. doi:10.1186/s12967-020-02691-4

10. Llovet JM, De Baere T, Kulik L, et al. Locoregional therapies in the era of molecular and immune treatments for hepatocellular carcinoma. Nat Rev Gastroenterol Hepatol. 2021;18(5):293-313. doi:10.1038/s41575-020-00395-0

11. Zheng Q, Fu Q, Xu J, et al. Transcription factor E2F4 is an indicator of poor prognosis and is related to immune infiltration in hepatocellular carcinoma. J Cancer. 2021;12(6):1792-1803. doi:10.7150/ jca.51616

12. Lu J, Li B, Xiong X, et al. RNA sequencing reveals the long noncoding RNA and mRNA profiles and identifies long non-coding RNA TSPAN12 as a potential microvascular invasion-related biomarker in hepatocellular carcinoma. Biomed Pharmacother. 2020;126:110111. doi:10.1016/j.biopha.2020.110111

13. $\mathrm{Gu} \mathrm{X}$, Zheng $\mathrm{Q}$, $\mathrm{Chu} \mathrm{Q}$, et al. HAND2-AS1: a functional cancer-related long non-coding RNA. Biomed Pharmacother. 2021;137:111317. doi:10.1016/j.biopha.2021.111317 
14. Yang H, Huo J, Li X. Identification and validation of a five-gene prognostic signature for hepatocellular carcinoma. World J Surg Oncol. 2021;19(1):90. doi:10.1186/s12957-021-02202-9

15. Dai Q, Liu T, Gao Y, et al. Six genes involved in prognosis of hepatocellular carcinoma identified by Cox hazard regression. BMC Bioinform. 2021;22(1):167. doi:10.1186/s12859-02104095-7

16. Huang H, Zhao J, Wang TY, et al. Species-Specific Deamidation of RIG-I Reveals Collaborative Action between Viral and Cellular Deamidases in HSV-1 Lytic Replication. mBio. 2021;12(2). doi:10.1128/mBio.00115-21
17. Bibi N, Parveen Z, Nawaz MS, et al. In Silico Structure Modeling and Molecular Docking Analysis of Phosphoribosyl Pyrophosphate Amidotransferase (PPAT) with Antifolate Inhibitors. Curr Cancer Drug Targets. 2019;19(5):408-416. doi:10.2174/15680096196 66181127115015

18. Zam W, Ali L. Immune checkpoint inhibitors in the treatment of cancer. Curr Clin Pharmacol. 2021;16. doi:10.2174/1574884 716666210325095022

19. Zhang J, Tian Q, Zhang M, et al. Immune-related biomarkers in triple-negative breast cancer. Breast Cancer. 2021;28(4):792-805. doi:10.1007/s12282-021-01247-8

\section{Publish your work in this journal}

The International Journal of General Medicine is an international, peer-reviewed open-access journal that focuses on general and internal medicine, pathogenesis, epidemiology, diagnosis, monitoring and treatment protocols. The journal is characterized by the rapid reporting of reviews, original research and clinical studies across all disease areas. The manuscript management system is completely online and includes a very quick and fair peer-review system, which is all easy to use. Visit http://www.dovepress.com/ testimonials.php to read real quotes from published authors. 